\title{
Changing Computer-Usage Behaviors: What Users Want, Use, and Experience
}

\author{
Mina Khan \\ MIT Media Lab \\ Cambridge, MA, USA \\ minakhan01@gmail.com
}

\author{
Kathryn Wantlin* \\ Harvard University \\ Cambridge, MA, USA \\ kathrynwantlin@college.harvard.edu
}

\author{
Zeel Patel ${ }^{*}$ \\ Harvard University \\ Cambridge, MA, USA \\ zeelpatel@college.harvard.edu
}

\author{
Elena Glassman \\ Harvard University \\ Cambridge, MA, USA \\ glassman@seas.harvard.edu
}

\author{
Pattie Maes \\ MIT Media Lab \\ Cambridge, MA, USA \\ pattie@media.mit.edu
}

\begin{abstract}
Computer-related behavior change is helpful for well-being. We conducted a survey to investigate three research questions and further inform the design of computer-related behavior change applications. RQ1: What do people want to change and why/how? RQ2: What applications do people use or have used, why do they work or not, and what additional support is desired? RQ3: What are helpful/unhelpful computer breaks and why? Our survey had 68 participants and three key findings. First, time management is a primary concern, but emotional and physical side-effects are also important. Second, site blockers, self-trackers, and timers are commonly used, but they are ineffective as they are easy-to-ignore and not personalized. Third, away-from-computer breaks, especially involving physical activity, are helpful, whereas on-screen breaks are unhelpful, especially when they are long, because they are not refreshing. We recommend personalized and closed-loop computer-usage behavior change support and especially encouraging off-the-computer computer breaks.
\end{abstract}

\section{CCS CONCEPTS}

- Human-centered computing $\rightarrow$ User studies.

\section{KEYWORDS}

need-finding, user survey, intervention, computer usage, behavior change, personalization

\section{ACM Reference Format:}

Mina Khan, Kathryn Wantlin, Zeel Patel, Elena Glassman, and Pattie Maes. 2021. Changing Computer-Usage Behaviors: What Users Want, Use, and Experience. In Asian CHI Symposium 2021 (Asian CHI Symposium 2021 ), May 8-13, 2021, Yokohama, Japan. ACM, New York, NY, USA, 8 pages. https://doi.org/10.1145/3429360.3468180

\footnotetext{
*Both authors contributed equally to this research.

Permission to make digital or hard copies of all or part of this work for personal or classroom use is granted without fee provided that copies are not made or distributed for profit or commercial advantage and that copies bear this notice and the full citation on the first page. Copyrights for components of this work owned by others than ACM must be honored. Abstracting with credit is permitted. To copy otherwise, or republish, to post on servers or to redistribute to lists, requires prior specific permission and/or a fee. Request permissions from permissions@acm.org.

Asian CHI Symposium 2021, May 8-13, 2021, Yokohama, Japan

(c) 2021 Association for Computing Machinery.

ACM ISBN 978-1-4503-8203-8/21/05 . .\$15.00

https://doi.org/10.1145/3429360.3468180
}

\section{INTRODUCTION}

Technology has become an intrinsic part of our lives, but excessive technology use is also connected to several physical and psychological problems [11, 18, 30,31]. Knowledge workers heavily rely on computers [17] and recently, there has been a significant increase in computer usage, especially during the COVID-19 pandemic [6].

There are several scales for accessing computer usage, e.g., computer use scale [27], attitudes toward computer usage scale [28], and compulsive internet use scale [24]. Research has been done to understand as well as modify computer and phone usage, e.g., encourage physical activity [3], and enable self-tracking [29] and better focus $[2,23]$. There has also been research on identifying user needs, e.g., self-monitoring [25] and productivity needs [10, 17], and what people consider to be work-breaks [9] and what breaks are helpful for productivity [9].

We investigate 3 research questions to further inform the design of computer-usage behavior change applications, including applications for encouraging computer-related breaks. RQ1. Computerrelated Behavior Change Needs and Desired Changes: What what do people want to change about their computer usage and why/how? RQ2. Currently-used Computer-usage Behavior Change Tools and Further Needs: What techniques or applications do people already use or have used for computer-related behavior change and why do they work or not work, and what kind of additional support do people require? RQ3. Computer-related Helpful and Unhelpful Breaks: What are helpful/unhelpful computer breaks and why are they are helpful/unhelpful?

\section{RELATED WORK}

Research has focused on both understanding and modifying computer and phone usage as well as inferring user states and contexts based on their phone and computer usage, especially for delivering interventions. While previous work focused on specific user needs, e.g., promoting productivity, physical activity, self-tracking, and focus, our work sheds light on the overall computer-usage behavior change needs of the users and highlights what applications and experiences are helpful/unhelpful for the users and why. Our related work is as follows.

\subsection{Understanding computer usage and user needs}

There are different scales for accessing computer usage, e.g., computer use scale [27], attitudes toward computer usage scale [28], and 
compulsive internet use scale [24]. Researchers have also used in situ studies to investigate computer-usage behavior change needs, e.g., self-monitoring [25] and break prompts that discourage sedentary behavior [20]. Researchers have also used diary studies and experience sampling to study more holistic scenarios, e.g., combining classic productivity with well-being [10], understanding personal productivity beyond work-related productivity [17], and understanding what makes smartphone use meaningful or meaningless [19]. Epstein et al., in particular, conducted a survey to identify what types of breaks, e.g., digital and biological breaks, people consider as breaks from work and what are the desirable qualities of a break, e.g., refreshing, relaxing [9]. Epstein et al.'s diary study focused on helpful breaks for productivity but not necessarily helpful and unhelpful breaks for overall user needs, e.g., physical and emotional health [9]. Our work aims extend this work by surveying the overall user needs, identifying what support works and does not work for users, and which computer breaks are helpful/unhelpful for overall user needs.

\subsection{Computer-usage self-tracking and behavior change applications}

There have been several applications to help users monitor and manage their phone and computer usage. While some applications enable passive self-tracking [1, 12, 29], others employ active interventions, e.g., for self-control on Facebook [21], for promoting mobility during work-breaks [3], for regulating phone usage $[15,16,26]$, and for blocking distractions to improve workplace focus and productivity [23]. Researchers have also studied different design choices, e.g., comparing goal-prompt versus removing newsfeed on Facebook [21], using physiological and location sensing for mobility prompts [3], comparing a point-of-choice prompt with an always-on progress bar to change sedentary behavior [32], giving feedback on interruption durations to discourages distractions and interruptions [2], and using lockout mechanisms $[15,16]$ or even nudge-like vibrations [26] for regulating phone usage. Researchers have also investigated individual differences in the effects of blocking workplace distractions [22]. However, researchers focus on specific needs, e.g., increasing productivity and reducing distractions or sedentary behaviors, not on overall user needs.

\subsection{Modeling user states and opportune moments}

Studies have monitored phone and computer usage, even combined with physiological data, to not only automatically recognize breaks and work activities [8], but also to model opportune moments for transitions and breaks at work for optimizing happiness and productivity [14]. There is also research to infer opportune moments for well-being messages on mobile phones, e.g., interventions for attention management [4] and for discouraging sedentary behavior [5]. We focus on surveying the helpful/unhelpful breaks and support needs of users to further define the design of computer-usage behavior change interventions.

\section{COMPUTER-USAGE BEHAVIOR CHANGE NEEDS SURVEY DESIGN}

We conducted an anonymous survey and recruited the participants using convenience and snowball sampling. We shared the survey via department email lists and social media, inviting the participants to share their 'computer-usage patterns and behavior change needs'. There were no explicit inclusion or exclusion criteria for the participants and the participants did not receive any compensation for the survey.

Participants We had 68 participants (35 males, 33 females; $\mu=$ 32.9 years, $\sigma=14.8$ years; 28 students, 39 full-time workers, 1 retired) from 9 countries - 35 from the United States (6 different states), 27 from Malaysia, 24 from the United Kingdom, 4 from Pakistan, 2 from Canada, 2 from India, 1 from France, 2 from Singapore, and 1 from Germany.

SuRvey Questions: We created our own survey since there was no preexisting survey to investigate our three research questions. We started with Likert scale questions to minimally survey the overall computer usage patterns (Q1-2) and broad problems categories (Q3) of the participants. We did not include full standardized computer usage surveys like CUS to keep our survey short. We then included open-ended questions (Q4-9) to survey the diverse and detailed experiences of our participants for each of our research questions - RQ1: Computer-usage behavior change needs (Q4) and specific desired changes (Q5); RQ2: Currently-used computerusage behavior change applications and if and why they work or do not work (Q6), and further-desired support (Q7); RQ3: User experiences with helpful computer breaks and why they are helpful (Q8), and similarly for unhelpful breaks (Q9). We iteratively developed the survey questions via peer review and expert review (5 experts and 5 peers) to ensure the validity and reliability of our questions. We also did 5 pilot surveys to further check validity and reliability. All survey questions are in Table 1.

DATA ANALYsis: For each of the open-ended questions (Q4-9), three researchers independently coded the responses and then collectively performed a thematic analysis of the responses. We performed inductive analysis and the 3 researchers iterated on the codes, themes, and categories for each question before finalizing them. We share the coded responses, themes, and also the top 50 words in each of the responses (excluding words repeated from the question).

\section{RESULTS}

We summarize below the results from our survey below for each of the research questions: RQ1. Computer-related Problems and Behavior Change Needs (Q4-5); RQ2. Currently-used Computer-usage Behavior Change Tools and Further Needs (Q6-7); RQ3. Computerrelated Helpful and Unhelpful Breaks (Q8-9). Also, we summarize the computer-usage patterns (Q1-Q2) and overall problems (Q3) below.

Q1, 2. Computer Usage Duration: Most participants reported '5-10 hours' of total computer usage. For 'Work/ Learning', the most common duration was '5-10 hours', and for 'Social Networking', 'Fun/Relaxation', and 'Miscellaneous', the most common duration was '0-2 hours' each. The detailed results are shown in Figure 1. 
Table 1: Survey questions about computer-usage patterns, behavior change needs, and helpful/unhelpful breaks

\begin{tabular}{|c|c|}
\hline $\mathbf{Q}$ & Survey Questions \\
\hline & Computer-Usage Patterns and Overall Problems \\
\hline 1 & How much time do you spend using your computer daily? 5 options: $0-2,2-5,5-10,10-15,>15$ hours \\
\hline 2 & How much do you use your computer/internet for the following (daily)? 4 categories and 5 options each: \\
\hline & Work/Learn, Social Network, Fun/Relax, Miscellaneous (Options: 0-2, 2-5, 5-10, 10-15, >15 hrs) \\
\hline 3 & How often do you experience the following side-effects due to your computer/internet usage? \\
\hline & $\begin{array}{l}5 \text { categories: Poor time management/distraction, Emotional stress, Physical discomfort, Social problems, } \\
\text { Financial problems; } 5 \text { single-select options for each category: Never, Rarely, Sometimes, Often, Always }\end{array}$ \\
\hline & RQ1. Computer-related Behavior Change Needs and Specific Desired Changes \\
\hline 4 & Is there anything you would like to change about your computer usage? Why and how? \\
\hline 5 & $\begin{array}{l}\text { Is there something you'd like to spend less time on or more time on? Please be specific, e.g., give examples. } \\
\text { RQ2. Currently-used Computer-usage Behavior Change Tools and Further Needs }\end{array}$ \\
\hline 6 & Is there anything you use or have used to manage your computer usage? Does it work? Why or why not? \\
\hline 7 & Is there something you would like to help you manage your computer usage? \\
\hline & RQ3. Computer-related Helpful and Unhelpful Breaks \\
\hline 8 & $\begin{array}{l}\text { Think of an example of a helpful computer break you took. (a) What activity did you do and how did it come } \\
\text { about? (b) How long was the break? (c) Why was it helpful? }\end{array}$ \\
\hline 9 & $\begin{array}{l}\text { Think of an example of an unhelpful computer break you took. (a) What was the break and how did it come } \\
\text { about? (b) How long was the break? (c) Why was it unhelpful? }\end{array}$ \\
\hline
\end{tabular}

Q3. Computer-Related Problems: Most participants 'Often' experienced 'Time management' problems, 'Sometimes' experienced 'Emotional stress' and 'Physical discomfort', and 'Never' experienced 'Social problems' and 'Financial problems' (though 'Sometimes' was a close second for 'Social problems'). The detailed results are shown in Figure 1.

\subsection{RQ1. Computer-related Problems and Behavior Change Needs (Q4-5)}

Q4. Desired Changes: 45 participants responded with specific desired changes (Q4), and we coded the responses into five categories - Reduce time drain (20), Reduce social media (10), Reduce physical side-effects (9), Reduce entertainment (3), and Improve device set-up (3). Q5. Desired Activities and Reasons: The participants mentioned several different specific desired activities (Q5), and we grouped the responses into 10 categories reflecting the underlying reasons for change - 'Better time management' (12), 'Feel emotionally better' (8), 'Reduce checking phone' (6), 'Better posture/reduce eye strain '(6), 'Less addictive scrolling' (4), etc. For both Q4 and Q5, time management, emotional well-being, and physical health were common themes. Figure 2 shows the coded responses for desired changes (Q4) and reasons for activity changes (Q5). Figure 4 shows the top 50 words in the responses to Q4 (left) and Q5 (right).

\subsection{RQ2. Currently-used Computer-usage Behavior Change Tools and Further Needs (Q6-7)}

Q6. Tools Used and their Efficacy: We received 22 responses about the tools used by the participants for computer-related behavior change. Site blockers were the most commonly used types of tools, followed by activity trackers, and then equally by selfplanning tools, Pomodoro-technique apps, and timers. However, most of the applications used by participants were not helpful, mostly because they were 'Easy to ignore' or 'Not appealing'. Reasons why some applications were helpful included 'helps fatigue', 'like tracking features', 'gamification', 'like complete blocker', and 'like variation'. Figure 2 (right) shows the categories of tools used, their efficacy, and the reasons behind their efficacy.

Q7. Further Support Desired: 53 participants responded - 11 did not know what they wanted, 5 did not need any, and rest had diverse suggestions. We created 17 categories for the responses, e.g., ergonomics solutions (6), reminders notifications (6), learned behavior and interventions (5), selective blockers (5), etc. The coded responses are in Figure 3.

Figure 5 shows the top 50 words in the responses to Q6 (left) and Q7 (right).

\subsection{RQ3. Computer-related Helpful and Unhelpful Breaks (Q8-9)}

Q8. Helpful Breaks and Reasons: We divided the responses into 16 categories. Some participants mentioned multiple breaks or one break that fell into multiple categories, e.g., housework also involved physical activity. The most common category of helpful breaks was physical activity (25), followed by housework (9), time in nature/outside (7), food/water breaks (7), eye exercises (3), family/friends time (3), watching videos (3), doing art (3), etc. Overall, most of the helpful breaks were away from the computer and the breaks were diverse, e.g., 'Nature/outside' was gardening for some and watching the sunset for others. The duration of helpful breaks was diverse: $<5 \mathrm{~min}(5), 5-15 \mathrm{~min}(8), 15-30 \mathrm{~min}$ (14), 30-60 $\mathrm{min}$ (9), 1 hour-1 day (4), >1 day (2). 41 participants mentioned the reasons for 'helpful' breaks, and we divided the responses into 13 categories - Physical breaks/ break from sitting (10), Mental break/ break from work (7), Screen break (6), Refreshing (5), Focus on something else (3), Feel good (2), Learning/ growth (2), etc. The results are in Figure 6. 

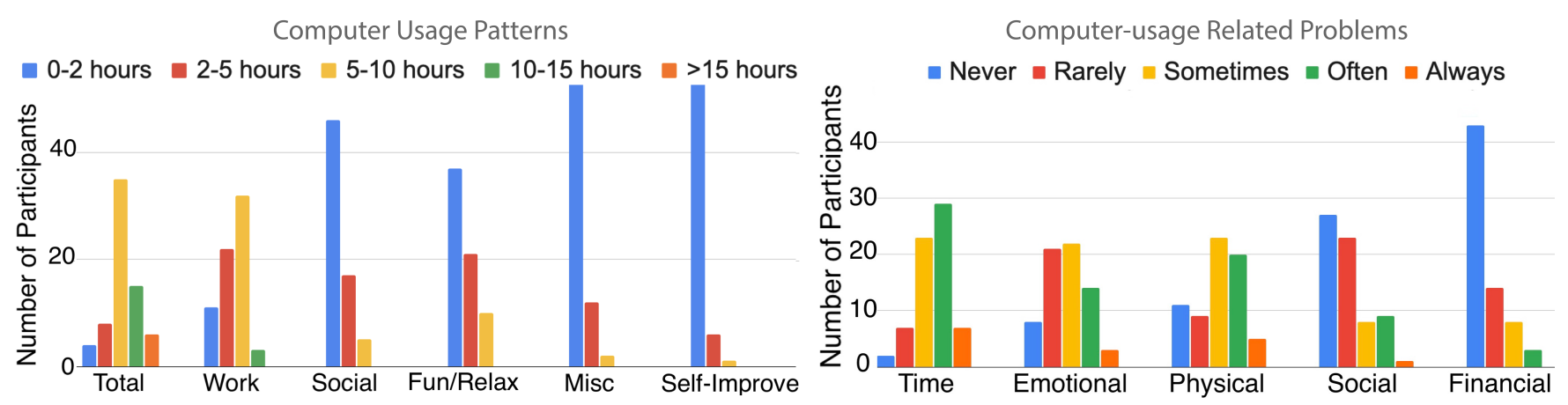

Figure 1: Likert scale responses. Left: Computer Usage Patterns (Q1, 2). Right: Computer-usage Problems (Q3)
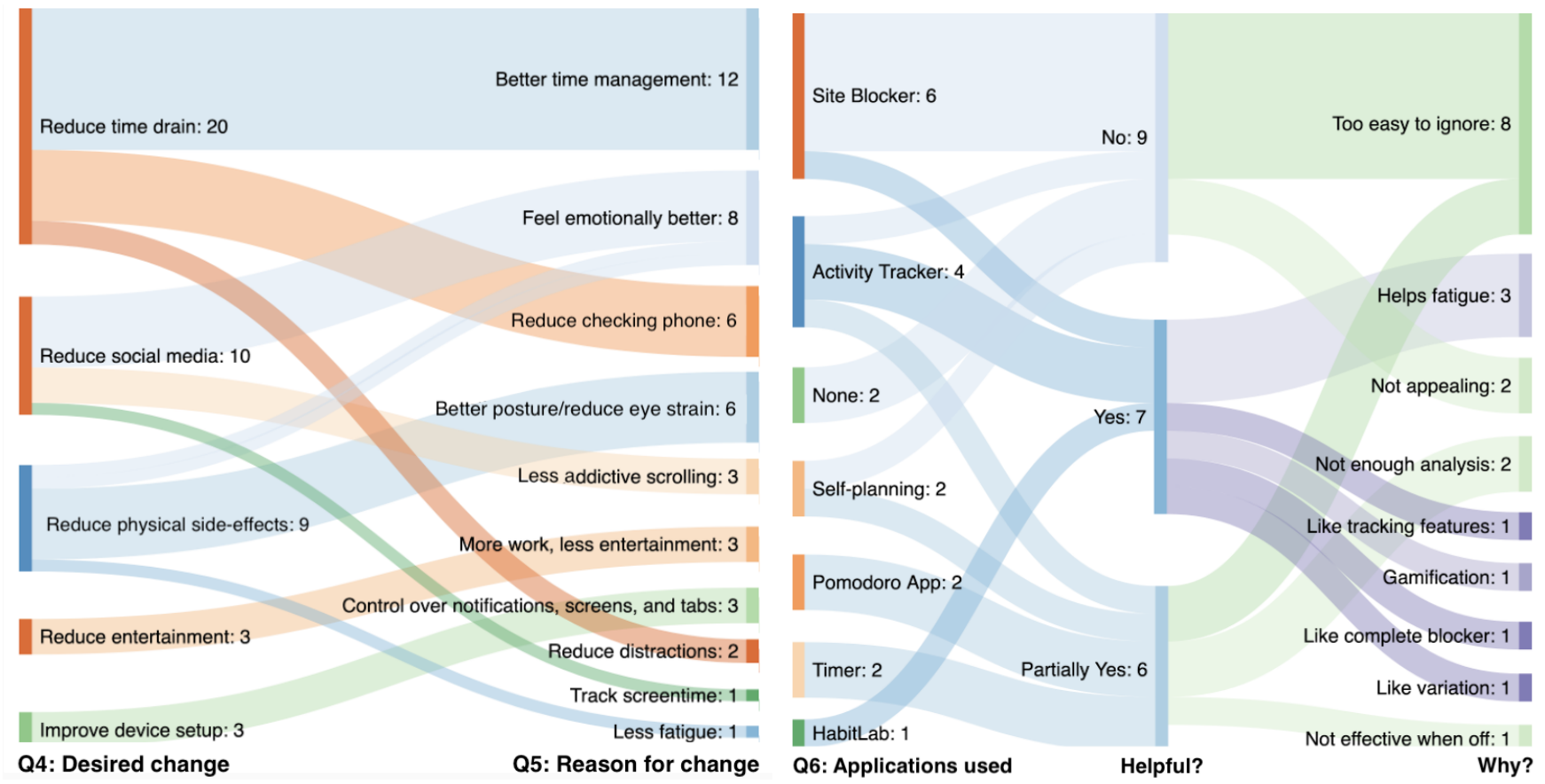

Figure 2: Coded responses for open-ended questions (Q4-6). Left: Desired computer-usage behavior change (Q4) and specific desired activities (Q5). Right: Currently used support for computer-usage behavior change and why they do or do not work (Q6)

Q9. Unhelpful Breaks and Reasons: We divided the responses into 15 categories, which had some overlaps, e.g., 'screen' category was also connected to 'phone', 'media' and 'web browsing', but because some participants explicitly mentioned only one, we characterized the responses to closest mentioned category. Most of the unhelpful breaks involved phone or computer screens, e.g., games (5), social media (5), web browsing (1), and videos (12), or long social interactions (6). 7 participants mentioned the duration of unhelpful breaks, and 6 of them reported more than 30-minute-long breaks: 15-20 $\min$ (1), 30-60 min (5), 1-3 hours (1). Finally, 31 participants mentioned why they found the break unhelpful, and we divided the responses into 13 categories - 'still screen' (5), 'not useful' (4), 'hard to refocus' (4), 'emotional distress' (4), 'not refreshing' (3), 'tiring' (2), 'too long' (2), 'distracting' (2), etc. The results are in Figure 7.
Figure 8 shows the raw top 50 words used in survey responses to Q8 (left) and Q9 (right).

\section{DISCUSSION AND RECOMMENDATIONS}

Previous computer-related behavior change work has focused on specific goals, e.g., improving productivity, focus and physical activity. We conducted a study to identify the overall user needs. We discuss the key findings, limitations, and recommendations of our computer-usage behavior change needs user survey below.

\subsection{Findings}

Most people spent between 5-10 hours on their computer (Q1, Q2), and time management, emotional problems, and physical discomfort are key concerns for people with respect to their computer 


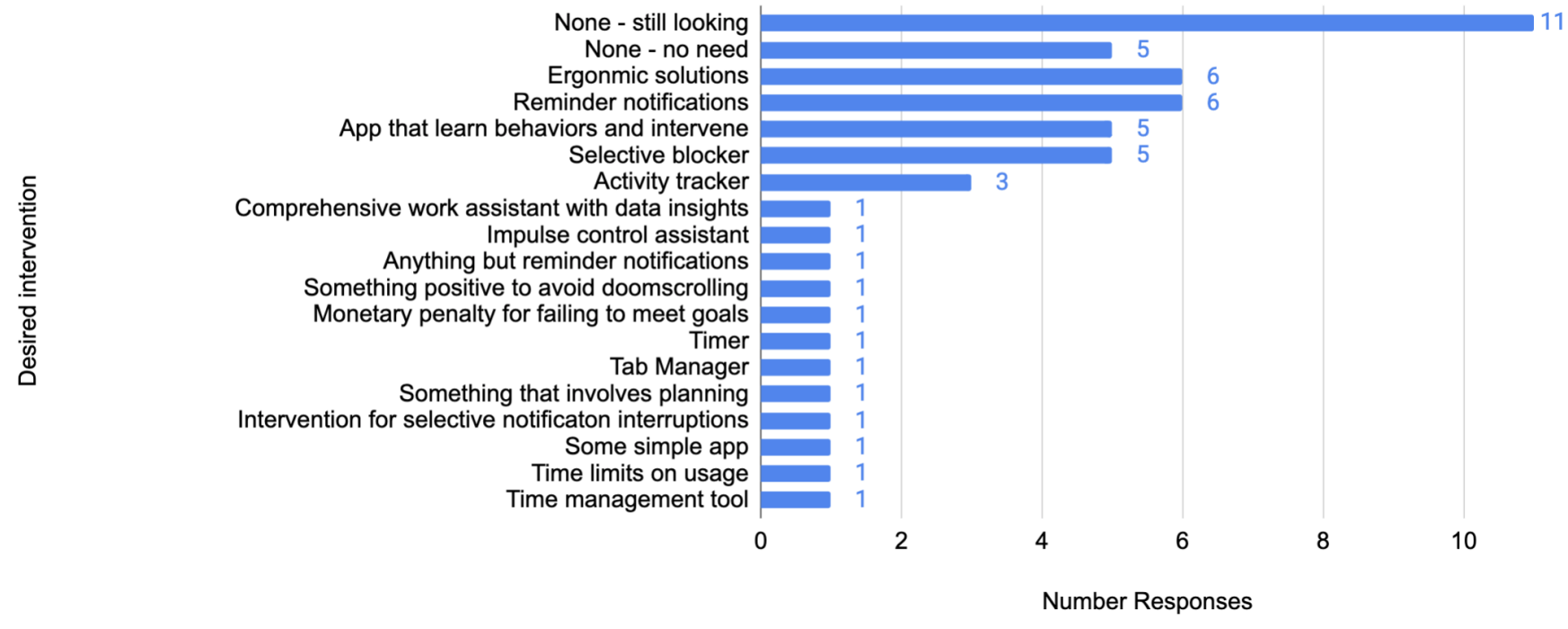

Figure 3: Open-ended responses for desired computer-usage behavior change support (Q7)

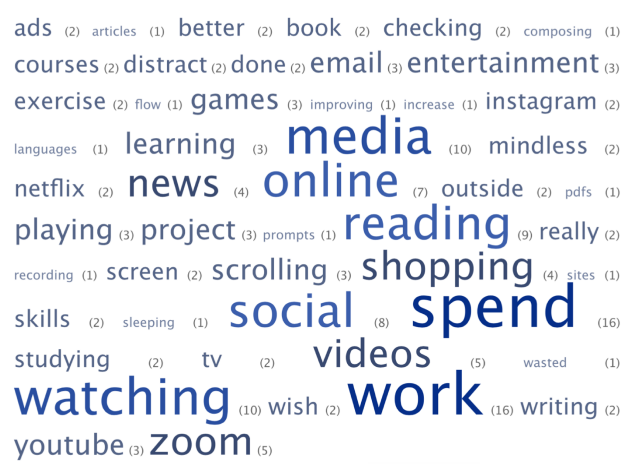

Figure 4: Top 50 words in open-ended responses. Left: Desired computer-usage change (Q4). Right: Specific activities change (Q5)

\section{Tried Applications and Efficacy}

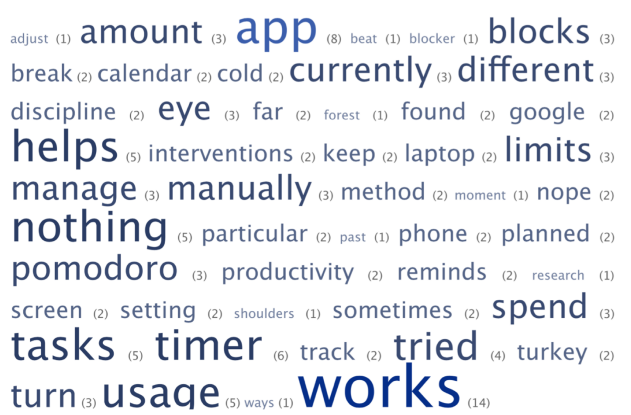

\section{Further Desired Support}

Figure 5: Top 50 words in open-ended responses. Left: Tried applications and their efficacy (Q6). Right: Further desired support (Q7) 


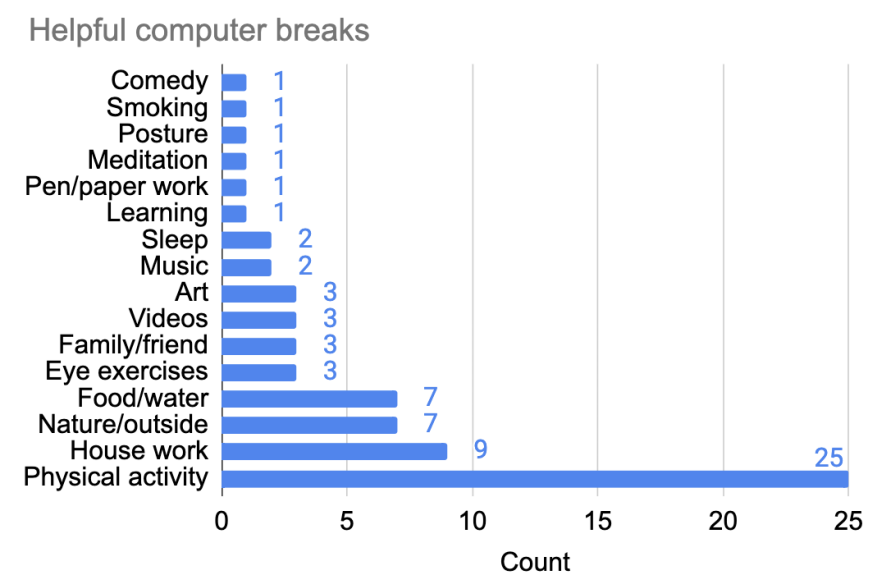

Why were they helpfu

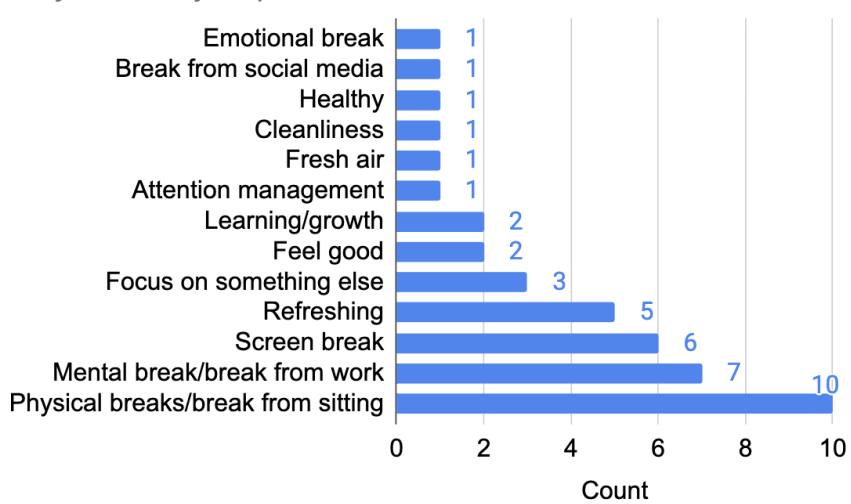

Figure 6: Q8. Coded open-ended responses for helpful computer-related breaks (left) and reasons why they were helpful (right)

Unhelpful Computer Breaks

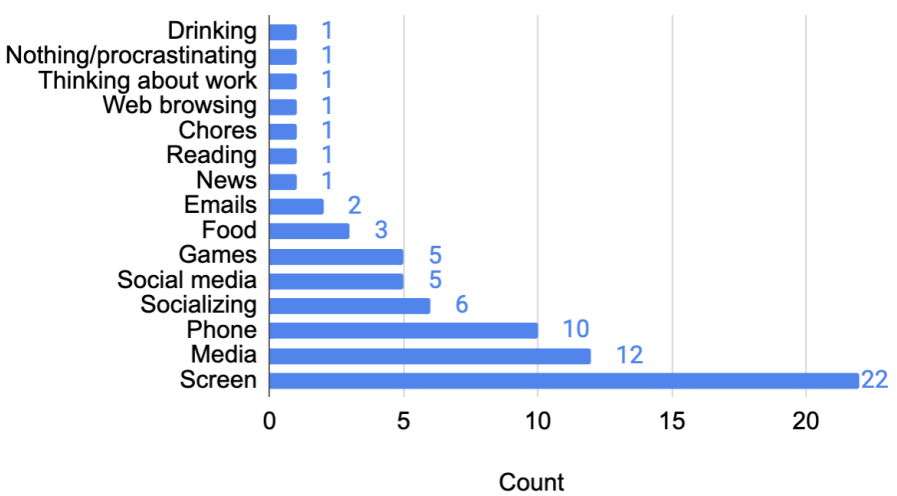

Why unhelpful

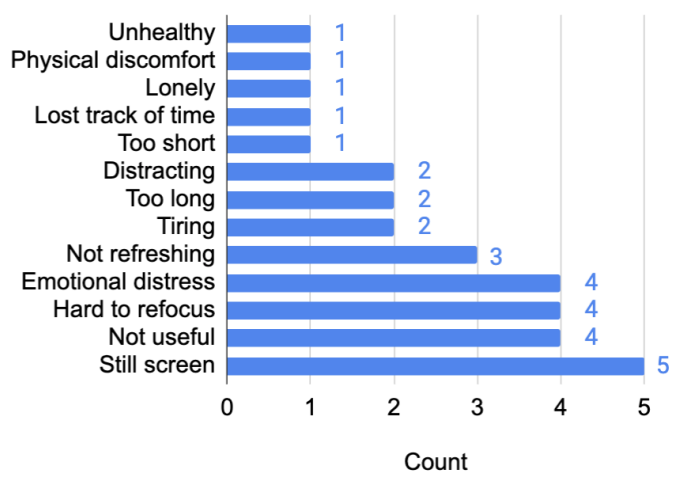

Figure 7: Q9. Open-ended responses for unhelpful computer-related breaks (left) and reasons why they were unhelpful (right)

\section{Helpful Computer Breaks}

\section{Unhelpful Computer Breaks}

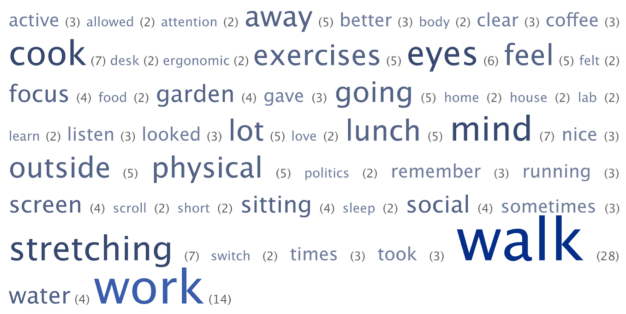

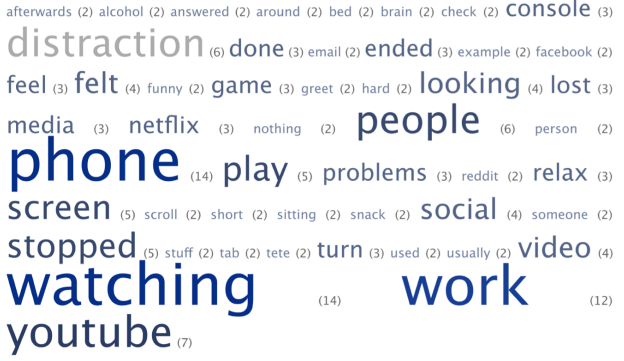

distraction (6) done (3) email (2) ended (3) example (2) facebook (2) feel (3) felt (4) funny (2) game (3) greet (2) hard (2) looking (4) lost (3) media (3) netflix (3) nothing (2) people (6) person (2) phone (14) play (5) problems (3) reddit (2) relax (3) stopped watching work as youtube

Figure 8: Top 50 words in responses to Q8: Helpful computer-related breaks (left) and Q9: Unhelpful computer-related breaks (right)

usage (Q3). We had three key findings from our survey. First, the participants wanted to reduce time drain, social media usage, entertainment, and physical side-effects (Q4) to better manage their time, emotions, and physical health, and also do less addictive scrolling and 'phone checking' (Q5). Users needs were, thus, have diverse and intertwined needs as they want to reduce their time drain, social media usage, and physical discomfort (Q4) to better manage their time and physical and emotional health (Q5). Second, people use site blockers, time management, and self-tracking applications, but many do not work well, especially because they are easy to ignore and are not designed for different user needs in different contexts (Q6). Thus, users want personalized interventions (e.g., something positive) in personalized contexts (e.g., selective blockers) and with personalized self-tracking insights (Q7). Third, away-from-screen 
breaks were usually helpful, especially when they were under 1 hour and involved physical or mental breaks (Q8), whereas onscreen breaks were not helpful and even tended to leave people exhausted and demotivated to work, especially when they were longer than 1 hour (Q9).

\subsection{Limitations}

We highlight three limitations of our work. First, we conducted our survey during the COVID 2019 pandemic and the computer-usage behavior change needs may be different than 'normal' times since most work was done virtually. However, given the increasing reliance on technology, our survey highlights the current and future needs for computer-usage behavior change. Second, our survey shows diverse participant needs and responses and does not explore one specific area, e.g., overcoming distractions or sedentary behavior. However, it is important to highlight and harness the diverse user needs and experiences to support real-life user needs. Third, we focus on user needs, but the user may not know what is best for them, e.g., user's perceived efficacy may be different from actual efficacy (Q6), and user reports on helpful/unhelpful breaks $(\mathrm{Q} 8,9)$ may be biased memories. Thus, it is important to be mindful of user needs and experiences, but then also test them via in situ and longitudinal studies. Overall, our survey is the first of its kind and further surveys and studies may be needed to validate our findings in different settings and with different users.

\subsection{Recommendations}

We have three recommendations. First, even though computerrelated behavior change has focused on disjoint goals like productivity, focus, and physical activity, computer-usage behavior change needs are diverse and interconnected - reducing distractions is connected to better time management and productivity, and even physical activity breaks are helpful for "better" computer usage (Q8). The three broad categories of user needs are time management, emotional wellbeing, and physical health, and it may help to provide holistic and personalizable support for user's diverse and interconnected user needs. Second, computer-usage behavior change support can be easy-to-ignore and users want personalized and closed-loop support. We recommend closed-loop behavior change support using reinforcement learning to monitor each user and provide better personalized and context-aware support. Third, off-the-screen breaks are more helpful than on-the-screen breaks. Instead of only restricting on-the-screen breaks, especially since restrictions can be stressful [23], it might help to replace onthe-screen draining breaks with off-the-screen refreshing breaks. Also, compulsive technology use is a problem [7] and technology use has a cognitive cost [13]. Thus, we recommend encouraging off-the-screen breaks to curb on-the-screen distractions and boost on-the-screen productivity.

\section{CONCLUSION}

Previous research on computer-related behavior change has focused on goals like productivity, focus, or physical activity, or on evaluating what users consider as computer breaks and what are helpful breaks for productivity and focus. We conducted a need-finding survey to investigate three key research questions with respect to people's computer-usage change: i. what do people want to change and why; ii. what do people currently use or have used and what is further desired; iii. what are helpful and unhelpful breaks and why.

Our findings show that user needs for computer-related behavior change are diverse and interconnected, e.g., the need for better time management is connected to better productivity and focus and even better physical activity as physical movement enables "better" overall computer usage experience. Many computer-usage-related behavior change applications do not work for the users as they are easy-to-ignore and also not personalized for users. Finally, off-thescreen breaks are, in general, helpful whereas on-the-screen breaks are not helpful.

Thus, user needs are interconnected and users need personalized, holistic, and closed-loop support. One way to offer personalized and closed-loop support is to use reinforcement learning to learn the best context-aware interventions for each user. Also, off-thecomputer breaks may be more helpful than on-the-computer breaks. We believe that our findings will inform the design of future behavior change applications for computer usage.

\section{REFERENCES}

[1] Gabriel Barata, Hugo Nicolau, and Daniel Gonçalves. 2012. AppInsight: what have I been doing?. In Proceedings of the International Working Conference on Advanced Visual Interfaces (AVI '12). Association for Computing Machinery, New York, NY, USA, 465-472. https://doi.org/10.1145/2254556.2254645

[2] Judith Borghouts, Duncan P. Brumby, and Anna L. Cox. 2020. TimeToFocus: Feedback on Interruption Durations Discourages Distractions and Shortens Interruptions. ACM Transactions on Computer-Human Interaction 27, 5 (Aug. 2020), 32:1-32:31. https://doi.org/10.1145/3396044

[3] Scott A Cambo, Daniel Avrahami, and Matthew L Lee. 2017. BreakSense: Combining physiological and location sensing to promote mobility during work-breaks. In Proceedings of the 2017 CHI Conference on Human Factors in Computing Systems. 3595-3607.

[4] Seyma Kucukozer Cavdar, Tugba Taskaya-Temizel, Mirco Musolesi, and Peter Tino. 2020. A Multi-perspective Analysis of Social Context and Personal Factors in Office Settings for the Design of an Effective Mobile Notification System. Proceedings of the ACM on Interactive, Mobile, Wearable and Ubiquitous Technologies 4, 1 (March 2020), 15:1-15:38. https://doi.org/10.1145/3381000

[5] Woohyeok Choi, Sangkeun Park, Duyeon Kim, Youn-kyung Lim, and Uichin Lee. 2019. Multi-Stage Receptivity Model for Mobile Just-In-Time Health Intervention. Proceedings of the ACM on Interactive, Mobile, Wearable and Ubiquitous Technologies 3, 2 (June 2019), 39:1-39:26. https://doi.org/10.1145/3328910

[6] J Clement. 2020. Coronavirus: Impact on Online Usage in the US-Statistics \& Facts. Hamburg: Statista (2020).

[7] Jeffrey A Clements and Randall Boyle. 2018. Compulsive technology use: Compulsive use of mobile applications. Computers in Human behavior 87 (2018), $34-48$.

[8] Elena Di Lascio, Shkurta Gashi, Juan Sebastian Hidalgo, Beatrice Nale, Maike E. Debus, and Silvia Santini. 2020. A Multi-Sensor Approach to Automatically Recognize Breaks and Work Activities of Knowledge Workers in Academia. Proceedings of the ACM on Interactive, Mobile, Wearable and Ubiquitous Technologies 4, 3 (Sept. 2020), 78:1-78:20. https://doi.org/10.1145/3411821

[9] Daniel A Epstein, Daniel Avrahami, and Jacob T Biehl. 2016. Taking 5: Workbreaks, productivity, and opportunities for personal informatics for knowledge workers. In Proceedings of the 2016 CHI Conference on Human Factors in Computing Systems. 673-684.

[10] Hayley Guillou, Kevin Chow, Thomas Fritz, and Joanna McGrenere. 2020. Is Your Time Well Spent? Reflecting on Knowledge Work More Holistically. In Proceedings of the 2020 CHI Conference on Human Factors in Computing Systems (CHI '20). Association for Computing Machinery, New York, NY, USA, 1-9. https: //doi.org/10.1145/3313831.3376586

[11] Jane M Healy. 1999. Failure to connect: How computers affect our children's mindsfor better and worse. Simon and Schuster.

[12] Donghan Hu and Sang Won Lee. 2019. ScreenTrack: Using Visual History for Selftracking Computer Activities and Retrieving Working Context. In The Adjunct Publication of the 32nd Annual ACM Symposium on User Interface Software and Technology (UIST '19). Association for Computing Machinery, New York, NY, USA, 44-46. https://doi.org/10.1145/3332167.3357110

[13] Sanghoon Kang and Terri R Kurtzberg. 2019. Reach for your cell phone at your own risk: The cognitive costs of media choice for breaks. Journal of behavioral 
addictions 8, 3 (2019), 395-403.

[14] Harmanpreet Kaur, Alex C. Williams, Daniel McDuff, Mary Czerwinski, Jaime Teevan, and Shamsi T. Iqbal. 2020. Optimizing for Happiness and Productivity: Modeling Opportune Moments for Transitions and Breaks at Work. In Proceedings of the 2020 CHI Conference on Human Factors in Computing Systems (CHI '20). Association for Computing Machinery, New York, NY, USA, 1-15. https://doi org/10.1145/3313831.3376817

[15] Jaejeung Kim, Hayoung Jung, Minsam Ko, and Uichin Lee. 2019. GoalKeeper: Exploring Interaction Lockout Mechanisms for Regulating Smartphone Use. Proceedings of the ACM on Interactive, Mobile, Wearable and Ubiquitous Technologies 3, 1 (March 2019), 16:1-16:29. https://doi.org/10.1145/3314403

[16] Jaejeung Kim, Joonyoung Park, Hyunsoo Lee, Minsam Ko, and Uichin Lee. 2019. LocknType: Lockout Task Intervention for Discouraging Smartphone App Use. In Proceedings of the 2019 CHI Conference on Human Factors in Computing Systems (CHI '19). Association for Computing Machinery, New York, NY, USA, 1-12. https://doi.org/10.1145/3290605.3300927

[17] Young-Ho Kim, Eun Kyoung Choe, Bongshin Lee, and Jinwook Seo. 2019. Understanding Personal Productivity: How Knowledge Workers Define, Evaluate, an Reflect on Their Productivity. In Proceedings of the 2019 CHI Conference on Human Factors in Computing Systems (CHI '19). Association for Computing Machinery, New York, NY, USA, 1-12. https://doi.org/10.1145/3290605.3300845

[18] Daria J Kuss and Halley M Pontes. 2018. Internet addiction. Vol. 41. Hogrefe Verlag.

[19] Kai Lukoff, Cissy Yu, Julie Kientz, and Alexis Hiniker. 2018. What Makes Smartphone Use Meaningful or Meaningless? Proceedings of the ACM on Interactive, Mobile, Wearable and Ubiquitous Technologies 2, 1 (March 2018), 22:1-22:26. https://doi.org/10.1145/3191754

[20] Yuhan Luo, Bongshin Lee, Donghee Yvette Wohn, Amanda L. Rebar, David E. Conroy, and Eun Kyoung Choe. 2018. Time for Break: Understanding Information Workers' Sedentary Behavior Through a Break Prompting System. In Proceedings of the 2018 CHI Conference on Human Factors in Computing Systems. Association for Computing Machinery, New York, NY, USA, 1-14. https://doi.org/10.1145 3173574.3173701

[21] Ulrik Lyngs, Kai Lukoff, Petr Slovak, William Seymour, Helena Webb, Marina Jirotka, Jun Zhao, Max Van Kleek, and Nigel Shadbolt. 2020. 'I Just Want to Hack Myself to Not Get Distracted' Evaluating Design Interventions for Self-Contro on Facebook. In Proceedings of the 2020 CHI Conference on Human Factors in Computing Systems. 1-15.

[22] Gloria Mark, Mary Czerwinski, and Shamsi T Iqbal. 2018. Effects of individual differences in blocking workplace distractions. In Proceedings of the $2018 \mathrm{CH}$
Conference on Human Factors in Computing Systems. 1-12.

[23] Gloria Mark, Shamsi Iqbal, and Mary Czerwinski. 2017. How blocking distractions affects workplace focus and productivity. In Proceedings of the 2017 ACM International foint Conference on Pervasive and Ubiquitous Computing and Proceedings of the 2017 ACM International Symposium on Wearable Computers. 928-934.

[24] G-J Meerkerk, Regina JJM Van Den Eijnden, Ad A Vermulst, and Henk FL Garretsen. 2009. The compulsive internet use scale (CIUS): some psychometric properties. Cyberpsychology \& behavior 12, 1 (2009), 1-6.

[25] Andre N. Meyer, Gail C. Murphy, Thomas Zimmermann, and Thomas Fritz. 2017. Design Recommendations for Self-Monitoring in the Workplace: Studies in Software Development. Proceedings of the ACM on Human-Computer Interaction 1, CSCW (Dec. 2017), 79:1-79:24. https://doi.org/10.1145/3134714

[26] Fabian Okeke, Michael Sobolev, Nicola Dell, and Deborah Estrin. 2018. Good vibrations: can a digital nudge reduce digital overload?. In Proceedings of the 20th International Conference on Human-Computer Interaction with Mobile Devices and Services (MobileHCI '18). Association for Computing Machinery, New York, NY, USA, 1-12. https://doi.org/10.1145/3229434.3229463

[27] Jan C Panero, David M Lane, and H Albert Napier. 1997. PART I: The Compute use Scale: Four Dimensions of how People use Computers. fournal of Educational Computing Research 16, 4 (1997), 297-315.

[28] Paula M Popovich, Karen R Hyde, Todd Zakrajsek, and Catherine Blumer. 1987. The development of the attitudes toward computer usage scale. Educational and psychological measurement 47, 1 (1987), 261-269.

[29] John Rooksby, Parvin Asadzadeh, Mattias Rost, Alistair Morrison, and Matthew Chalmers. 2016. Personal Tracking of Screen Time on Digital Devices. In Proceedings of the 2016 CHI Conference on Human Factors in Computing Systems (CHI '16). Association for Computing Machinery, New York, NY, USA, 284-296. https://doi.org/10.1145/2858036.2858055

[30] Noha Soliman Elserty, Nesma Ahmed Helmy, and Khaled Mohmed Mounir. 2020. Smartphone addiction and its relation to musculoskeletal pain in Egyptian physical therapy students. European fournal of Physiotherapy 22, 2 (2020), 70-78.

[31] Aaseer Thamby Sam Subramani Parasuraman, Stephanie Wong Kah Yee, Bobby Lau Chik Chuon, and Lee Yu Ren. 2017. Smartphone usage and increased risk of mobile phone addiction: A concurrent study. International journal of pharmaceutical investigation 7, 3 (2017), 125.

[32] Yunlong Wang and Harald Reiterer. 2019. The Point-of-Choice Prompt or the Always-On Progress Bar? A Pilot Study of Reminders for Prolonged Sedentary Behavior Change. In Extended Abstracts of the 2019 CHI Conference on Human Factors in Computing Systems (CHI EA '19). Association for Computing Machinery, New York, NY, USA, 1-6. https://doi.org/10.1145/3290607.3313050 